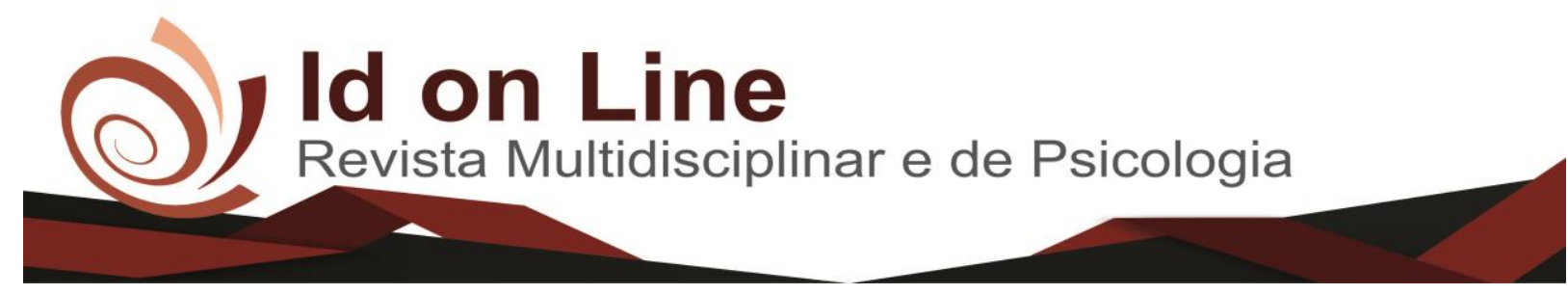

Artigo

\title{
Cobertura Vacinal do HPV para Adolescentes: Desafios e Possibilidades
}

\author{
Fernanda de Brito Pereira ${ }^{l}$; Érika Pereira de Souza $a^{2}$
}

\begin{abstract}
Resumo: A vacinação de adolescentes contra o Papiloma Vírus Humano - HPV é uma estratégia do Ministério da Saúde junto como o Programa Nacional de Imunização, que tem como público alvo meninas entre 9 a 14 anos e meninos de 12 a 13 anos, possuindo como porta de entrada as escolas e/ou Unidade Básica de Saúde. A vacinação tem por objetivo prevenir a incidência de novos casos de câncer do Colo do Útero e câncer de Pênis. Diante do exposto, o presente estudo tem como objetivo analisar a cobertura vacinal do HPV em adolescentes do sexo feminino e masculino no município de Ibiassucê, localizado no sudoeste da Bahia. Trata-se de uma pesquisa exploratória, revisão bibliográfica e quantitativa do tipo descritiva. Os dados foram coletados do Departamento de Informática do Sistema Único de Saúde (DATASUS).
\end{abstract}

Palavras-chave: Vacina; Doenças Sexualmente Transmissíveis; Escola; Papiloma vírus Humano;

\section{HPV Vaccinal Coverage for Adolescents: Challenges and Possibilities}

\begin{abstract}
The vaccination of adolescents against human papillomavirus (HPV) is a strategy of the Ministry of Health along with the National Program of Immunization, which has as public target girls between 9 to 14 years and boys from 12 to 13 years old, possessing as a gateway schools and / or Basic Health Unit. Vaccination aims to prevent the incidence of new cases of cervical cancer and penile cancer. In view of the above, the present study aims to analyze the HPV vaccination coverage in female and male adolescents in the municipality of Ibiassucê, located in the southwest of Bahia. This is an exploratory research, bibliographical and quantitative review of the descriptive type. Data were collected from the Department of Informatics of the Unified Health System (DATASUS).
\end{abstract}

Keywords: Vaccine; Sexually Transmitted Diseases; School; Human Papilloma virus;

\section{Introdução}

No ano de 2014, o ministério da saúde através do Programa Nacional de Imunizações (PNI), implementou a vacina contra o Papiloma Vírus Humano (HPV) no calendário de vacinação para meninas, disponíveis no Sistema Único de Saúde (SUS). A quarta causa de morte no Brasil em mulheres são as neoplasias do câncer do colo do útero (BRASIL, 2014).

\footnotetext{
${ }^{1}$ Graduação em Enfermagem pela faculdade Independente do Nordeste - FAINOR. Contato: fepereira00@ hotmail.com;

${ }^{2}$ Graduada em Enfermagem pela Universidade Federal da Bahia (UFBA). Especialista em Terapia Intensiva. Mestre em Ciências Fisiológicas. Doutoranda do Programa Multicêntrico de Pós Graduação em Ciências Fisiológicas pela Universidade Federal da Bahia.
} 
Dessa forma, surgiu a estratégia de rastreamento e a vacinação como uma forma promissora para prevenção de novos casos.

O Ministro da saúde, no uso das atribuições que lhe confere o inciso II, parágrafo único do art. 87 da Constituição Federal, considerando a Portaria no 3.124/GM, de 7 de dezembro de 2006, e considerando a conclusão dos trabalhos realizados pelo referido Grupo de Trabalho que reforça a necessidade de implementar ações de controle do câncer do Colo do Útero, visando a redução da incidência desse tipo de câncer na população, resolveu: Art. $1^{\circ}$ Instituir Comitê Permanente com a finalidade de acompanhar e monitorar a implementação de ações relativas à Vacina contra Papiloma Vírus Humano HPV (BRASIL, 2007).

O Papiloma vírus Humano (HPV) é umas das Infecções Sexualmente Transmissíveis (IST's) mais comuns, sendo capaz de causar o câncer do colo útero, pênis, garganta, vulva, vagina, ânus e as verrugas genitais. Dentre os mais de 200 tipos de HPV, os tipos virais mais comuns são o 6 e o 11 que são causadores das verrugas anogenitais e o 16 e 18, considerados os percussores dos carcinomas genitais (MACHADO, 2016).

As vacinas disponíveis atualmente são profiláticas, apresentando uma alta eficiência na indução da produção de anticorpos específicos, sendo os tipos virais $6,11,16$ e 18 . No ano de 2014 iniciou a campanha de vacinação nas escolas e em Unidades de Saúde da Família do SUS, com objetivo de distribuir três doses (0, 6 e 60) entre meninas de 11 a 13 anos. Em 2015, meninas a partir dos 9 anos foram incluídas na faixa etária de vacinação (KAVATI, 2017; QUEVEDO, 2016).

Em 2017, foram incluídas no calendário de vacinação as meninas que chegaram aos 14 anos que nunca receberam a vacina ou que não completaram as duas doses. Meninos na faixa etária de 12 a 13 anos. E a cada ano, a meta será inserir uma nova faixa etária em ordem decrescente (BOGAZ, 2016).

Sabe-se que as estratégias de prevenção primária são as informações e diálogo constante com os filhos, pactuado com os pais, professores e profissionais de saúde, divulgação e ensino de como usar o preservativo e outros métodos. Ter a escola como um ambiente na qual os adolescentes permanecem a maior parte do dia. Por este motivo a escola foi escolhida como cenário para a vacinação, pois terá maior índice de cobertura vacinal (CARNEIRO, 2014). 
A prevenção secundária tem como objetivo diagnosticar as doenças sexualmente transmissíveis. O tratamento se dá a partir de cada patologia, no qual permite o controle das infecções (CARNEIRO, 2014).

Após conhecimento sobre a vacina espera-se que ocorra uma aceitação dos pais, e autorização dos mesmos para o processo de vacinação dos filhos, contribuindo assim para que os filhos recebam as duas doses. Em contrapartida a recusa dos responsáveis se pautou nos fatores como crenças religiosas, e que a vacina poderia induzir o início de atividade sexual (SOUZA, 2015; ZANINI, 2015).

A importância da cobertura vacinal em adolescentes tem como finalidade, que eles possam chegar à fase adulta com menor risco de contrair o vírus do HPV, que é responsável pelo desenvolvimento do câncer do colo de útero, vulva, ânus, garganta e pênis. Sendo que o câncer do colo de útero ocupa a quarta posição de morte na população feminina (BOGAZ, 2016; BRASIL, 2014).

Dessa forma, devida à importância da cobertura vacinal, justifica-se a necessidade da realização deste trabalho, cujo objetivo é analisar a cobertura vacinal do HPV em adolescentes no município de Ibiassucê, localizado no sudoeste da Bahia e identificar a taxa de cobertura vacinal.

\section{Metodologia}

A presente pesquisa possui um caráter exploratório com abordagem quantitativa, pois trata-se de um estudo da cobertura vacinal do HPV descritivo, cujos dados foram obtidos por meio de consulta ao DATASUS (Departamento de Tecnologia da Informação a Serviço do SUS) e ao aplicativo de tabulação genérico (TABNET), onde contém informações que serviram para subsidiar a formulação e avaliação das políticas, planos e programas de saúde, facilitando o processo de tomada de decisões, com vistas a contribuir para a melhoria da situação de saúde da população através dos dados de maneira secundária.

O objeto de estudo teve como foco adolescentes meninas de 9 a 14 anos e meninos de 12 a 13 anos conforme o Ministério da Saúde, através dos dados fornecidos pelo DATASUS e das notificações entre os anos de 2014 a 2017 do município de Ibiassucê - Bahia. 
O aplicativo TABNET é um tabulador genérico de domínio público que permite organizar dados de forma rápida conforme a consulta que se deseja tabular. Foi desenvolvido pelo DATASUS para gerar informações das bases de dados do Sistema Único de Saúde.

Ademais, o presente estudo identifica-se como pesquisa bibliográfica desenvolvida mediante o estudo de teses de doutorado, dissertações de mestrado, artigos científicos, revistas e jornais especializados, livros, anais de congressos e em sites da web. A pesquisa bibliográfica representa um importante instrumento utilizado para a construção de um processo de investigação (LAKATOS; MARCONI, 2011).

Ibiassucê faz limite com quatro cidades e apresenta um alto índice de atendimento na rede do Sistema Único de Saúde. Possui economia como agropecuária e comércio, apresentando um PIB (Produto Interno Bruto) de 63.278,000 milhões por ano, segundo o IBGE 2014.

Foi utilizado um roteiro para a coleta de dados do DATASUS para a construção do perfil da cobertura vacinal do HPV no município de Ibiassucê - Bahia, analisados anualmente, de 2014 a 2017. Inicialmente foi pesquisado o número de doses aplicadas e a cobertura vacinal no total em Ibiassucê e foi confeccionada uma tabela do programa Microsoft Office Excel 2010® para tabulação dos resultados divididos por sexo, idade, e doses aplicadas.

Por se tratar de um banco de domínio público, não foi necessário submeter o projeto ao Comitê de Ética em Pesquisa, atendendo aos aspectos éticos constantes na Resolução nº466 do ano 2012 pertencente ao CNS- Conselho Nacional de Saúde.

\section{Resultados e Discussão}

Conforme os resultados obtidos pelo DATA SUS, a tabela 1 pode-se observar os dados das doses aplicadas em adolescentes no município do sudoeste da Bahia entre os anos de 2014 a 2017. Em 2014, ano da implantação da portaria da vacina, houve um número maior de doses aplicadas (465 doses) quando comparada com ano de 2017 que dá continuidade ao programa. No ano de 2014, o maior contingente de aplicação foi em adolescentes de 12 anos de idade, haja vista que essa vacina é aplicada entre 11 e 12 anos, sendo que o maior índice de doses aplicadas foi em meninas de 12 anos. O número de doses aplicadas foi de 105, correspondentes a D1 (Primeira dose).

No ano subsequente, 2015, o número maior de aplicações foi em crianças com 9 e 10 anos. Estes dados foram obtidos no ano que ocorreu uma redução na idade para aplicação, 
correspondente a meninas de 9 anos. Sendo que 80 crianças do sexo feminino receberam a D1. Em 2016 houve uma diminuição da quantidade de doses aplicadas em D1. Já em 2017, mesmo não tendo os dados em sua totalidade, não houve a finalização do ano, o mesmo apresentou um menor número de doses aplicadas em D1, o que pode ser justificado pela alta cobertura realizada nos anos anteriores. O total de doses aplicadas entre 2014 a 2017 foram de 1.020, sendo a quantidade de D1 foi 543 doses.

Fator que justifica a diminuição de doses aplicadas foi que a partir da segunda fase da campanha tornou-se possível aos municípios escolher se a vacinação ocorreria no ambiente das escolas públicas e privadas ou nas Unidades Básicas de Saúde (UBS). Sendo assim, muitos municípios optaram por efetuarem a vacinação somente nas UBS (OLIVEIRA, 2015).

É importante que os adolescentes recebam o esquema completo da vacina contra o HPV o mais precocemente possível, de preferência antes de se tornarem sexualmente ativos. $\mathrm{O}$ adolescente que recebe corretamente o esquema vacinal apresenta uma eficácia de $98 \%$ (ALMEIDA, 2014; BRASIL, 2015).

E de extrema importância salientar sobre a necessidade da segunda dose para que se tenha reforço e continuidade do estabelecimento de uma resposta imune adequada. Por mais que ao receber uma dose, há uma produção de anticorpos, esta não é suficiente para obtenção de uma resposta imune satisfatória.

$\mathrm{Na}$ análise dos dados ao comparar o município de Rolim de Moura - RO com Ibiassucê - BA, pode-se observar que em Rolim de Moura aplicou 508 doses em meninas de 9 a 11 anos no ano de 2015. Já em Ibiassucê foram 202 doses aplicadas para as meninas de 9 a 11 anos, referente ao mesmo ano 2015. Isso mostra um número baixo para a D1 da vacina no município de Ibiassucê. Essa baixa adesão em Ibiassucê pode está relacionada ao déficit de divulgação sobre a vacina, e o receio dos responsáveis a respeito da imunização, que é visto como incentivo à iniciação sexual (MACHADO, 2016).

Tabela 1- Dados das doses aplicadas em crianças do sexo feminino em um município do sudoeste da Bahia entre os anos de 2014 a 2017.

\begin{tabular}{llllll}
\hline IMUNO & 2014 & 2015 & 2016 & 2017 & TOTAL \\
\hline TOTAL & 465 & 395 & 89 & 71 & 1.020 \\
HPV Quadrivalente D1 9 anos & - & 80 & 39 & 23 & 142 \\
HPV Quadrivalente D2 9 anos & - & 37 & 16 & 16 & 69 \\
HPV Quadrivalente D1 10 anos & - & 59 & 14 & 1 & 74 \\
HPV Quadrivalente D2 10 anos & - & 70 & 10 & 20 & 100 \\
HPV Quadrivalente D1 11 anos & 66 & 63 & 1 & - & 130 \\
\hline
\end{tabular}




\begin{tabular}{llllll}
\hline HPV Quadrivalente D2 11 anos & 31 & 58 & 3 & 1 & 93 \\
HPV Quadrivalente D1 12 anos & 105 & 1 & - & - & 106 \\
\hline HPV Quadrivalente D2 12 anos & 81 & 26 & 3 & 3 & 113 \\
HPV Quadrivalente D1 13 anos & 87 & 1 & - & 3 & 91 \\
HPV Quadrivalente D2 13 anos & 95 & - & 3 & 4 & 102 \\
HPV Quadrivalente D1 14 anos & - & - & - & - & - \\
\hline
\end{tabular}

Fonte: Programa Nacional de Imunizações

A tabela 2 demonstra que 2017, foram inseridos os meninos de 12 e 13 anos de idade no calendário de vacinação, pois a nota informativa Nº2-SEI/2017-CGPNI/DEVIT/SVS/MS implementou as doses de vacina quadrivalente em meninos, haja visto pela necessidade de reduzir o índice de câncer de pênis, anal e boca, pois alguns adolescentes tem uma vida sexualmente ativa, e pode transmitir o vírus. Na tabela 2, foram aplicadas 84 doses. A maior quantidade de doses aplicadas foi em meninos de 13 anos. Sendo que os dados obtidos das doses aplicadas em meninos foram inferiores, quando comparado à primeira aplicação em meninas da mesma faixa etária em 2014.

$\mathrm{Na}$ Bahia foram aplicadas 85.166 doses em meninos nessa faixa etária, que corresponde à primeira dose. Apesar de todos os esforços para imunização dos adolescentes, os números de doses aplicadas continuam abaixo da meta estabelecida pelo governo. A adolescência é um período de transição que apresenta várias dificuldades, sendo uma delas de frequentar as unidades de saúde (DATASUS; COELHO, 2017).

A vacinação é uma prevenção primária e proteção específica que visa reduzir o surgimento de novos casos de doenças, assim há uma resposta imunológica que previne as reinfecções pelos vírus contidos nas vacinas por um longo período de tempo (ALMEIDA, 2014).

Tabela 2- Dados das doses aplicadas em crianças do sexo masculino no município do sudoeste da Bahia no ano de 2017.

\begin{tabular}{lll}
\hline IMUNO & 2017 & TOTAL \\
\hline TOTAL & 84 & 84 \\
HPV Quadrivalente D1 12 anos & 36 & 36 \\
HPV Quadrivalente D1 13 anos & 48 & 48 \\
\hline
\end{tabular}

Fonte: Programa Nacional de Imunizações

Na tabela 3, percebe-se que em 2014, ano de implementação da vacina, houve uma maior cobertura vacinal, que foi de $163,79 \%$ correspondente à segunda dose aplicada em meninas de 13 anos. No ano subsequente 2015, a cobertura vacinal em crianças de 9 anos foi 
de $114,08 \%$ na primeira dose. Ano que inclui essa nova faixa etária. Podemos observar que a cobertura foi satisfatória.

Em 2016 nota-se que houve uma redução na cobertura vacinal, ao comparado com o ano anterior. Sendo que a cobertura vacinal na faixa etária de 9 anos foi de 54,93\% que corresponde a primeira dose.

Neste ano de 2017, ano da continuação do esquema vacinal, dessa forma destaca-se que houve uma redução na cobertura vacinal, comparando desde a implantação da vacina em meninas de 9 anos. A cobertura vacinal foi de 48,94\% representa a primeira dose em meninas de 9 anos.

No ano que implementou a vacina, teve como cenário as escolas e Unidades de Saúde da Família do SUS. E ter a escola como cenário, sendo que é local onde os adolescentes estão em maior parte do dia, e isso contribui para ter uma cobertura vacinal excelente (QUEVEDO, 2016).

Uma das causas para a baixa cobertura vacinal em adolescente é a oportunidade perdida de vacinação, como consequência de não ter procurado o serviço de saúde, demostrando que há uma necessidade da equipe de saúde realizar atividades que envolvam a vacinação. Dessa forma ter a escola junto a Estratégia de Saúde da Família podendo abranger mais adolescentes, onde atingiria um índice maior de público alvo como também forneceria informações a respeito de transmissões do HPV (MACHADO, 2016).

Tabela 3 - Dados da Cobertura Vacinal em adolescentes do sexo feminino no município do sudoeste da Bahia no ano de 2017.

\begin{tabular}{|c|c|c|c|c|}
\hline IMUNO & 2014 & 2015 & 2016 & 2017 \\
\hline HPV Quadrivalente D1 9 anos & - & $114,08 \%$ & $54,93 \%$ & $48,94 \%$ \\
\hline HPV Quadrivalente D2 9 anos & - & $104,23 \%$ & $22,54 \%$ & $34,04 \%$ \\
\hline HPV Quadrivalente D1 10 anos & - & $84,29 \%$ & $20,00 \%$ & $2,13 \%$ \\
\hline HPV Quadrivalente D2 10 anos & - & $100 \%$ & $14,29 \%$ & $42,55 \%$ \\
\hline HPV Quadrivalente D1 11 anos & $102,86 \%$ & $90 \%$ & $1,43 \%$ & - \\
\hline HPV Quadrivalente D2 11 anos & $97,14 \%$ & $82,86 \%$ & $4,29 \%$ & $2,13 \%$ \\
\hline HPV Quadrivalente D1 12 anos & $152,17 \%$ & - & - & - \\
\hline HPV Quadrivalente D2 12 anos & $118,84 \%$ & $75,36 \%$ & $4,35 \%$ & $6,52 \%$ \\
\hline HPV Quadrivalente D1 13 anos & $150 \%$ & - & - & $6,38 \%$ \\
\hline HPV Quadrivalente D2 13 anos & $163,79 \%$ & - & $4,29 \%$ & $8,51 \%$ \\
\hline HPV Quadrivalente D1 14 anos & - & - & - & - \\
\hline
\end{tabular}

Fonte: Programa Nacional de Imunizações 
Na tabela 4 demostra a cobertura vacinal neste ano 2017 para meninos, em que houve uma cobertura de 87,27\% para adolescentes de 13 anos na primeira dose, em comparação com as meninas da mesma idade referente ao ano de 2014, percebe que os meninos tiveram um percentual inferior.

Tabela 4 - Dados da Cobertura Vacinal em adolescentes do sexo masculino no município do sudoeste da Bahia no ano de 2017.

\begin{tabular}{lll}
\hline IMUNO & 2017 & TOTAL \\
\hline HPV Quadrivalente D1 12 anos & $\mathbf{6 6 , 6 7 \%}$ & $\mathbf{6 6 , 6 7 \%}$ \\
HPV Quadrivalente D1 13 anos & $\mathbf{8 7 , 2 7 \%}$ & $\mathbf{8 7 , 2 7 \%}$ \\
\hline
\end{tabular}

Fonte: Programa Nacional de Imunizações

No estado de Goiás a cobertura vacinal em meninos de 12 anos foi de 16,34\% e 13 anos de 14,07\%. Observa-se que a cobertura vacinal está inferior em relação à Ibiassucê (SECRETÁRIA DE SAÚDE, 2017).

A meta do Ministério da Saúde é de $80 \%$ em todo território nacional, entretanto no mês de janeiro a junho de 2017 apenas 16,5\% dos meninos foram imunizados na faixa etária de 12 a 13 anos. Em contrapartida o município de Ibiassucê mostrou uma alta cobertura vacinal, em relação ao Brasil (COELHO, 2017).

Desta forma, a vacinação em 2017 seguirá estratégia mista, buscando alta participação das escolas pelo menos no primeiro mês de vacinação. Portanto estratégias de vacinação precisam ser reforçadas para alcançar melhores coberturas vacinais.

\section{Considerações Finais}

Analisando os dados do DATA SUS e dos autores citados acima, conclui-se que no início da campanha de vacinação do HPV, houve uma alta cobertura vacinal, sendo a utilização da escola como ponto estratégico para aplicação das vacinas em adolescentes.

Percebe-se que nos anos posteriores, teve uma redução na cobertura vacinal. Os fatores que influenciaram nos baixos resultados de adesão, foi à mudança do local estratégico, escola, para a Unidade Básica de Saúde. No entanto há uma necessidade que todas as doses sejam aplicadas no ambiente escolar. 
O objetivo da vacinação no Sistema Único de Saúde é reduzir o impacto do vírus no desenvolvimento de câncer do Colo do Útero, pênis, garganta, ânus. Para que se tenha uma geração imunizada contra o HPV, e assim diminuindo a incidência de novos casos de câncer. Contudo, a imunização não exclui o uso de preservativos nas relações sexuais e ações de rastreamento para o câncer do colo do útero, como a realização do exame preventivo de Papanicolaou em mulheres e Peniscopia nos homens.

Para que se tenha uma maior adesão dos adolescentes na vacinação contra o HPV, é necessário a realização de palestras e divulgação midiática sobre a importância da vacina como meio de prevenção. Os profissionais de saúde podem se unir com equipe pedagógica para a realização de palestras nas escolas, com o objetivo de informar os adolescentes e os responsáveis, sobre o Papiloma Vírus Humano, e como consequência alcançar o maior número de adolescentes imunizados.

\section{Referências}

ALMEIDA, G. C. P.; CAVEIÃO, C. Vacina profilática para o Papiloma Vírus Humano: Desafios para saúde pública. Revista Saúde e Desenvolvimento | vol.5 n.3 | jan/jun 2014. Disponível em <http://www.uninter.com/revistasaude/index.php/saudeDesenvolvimento/article/download/29 5/200> Acesso em: 27 Março 2017.

BOGAZ, C.; AMORIM, A.C. "Meninos também serão vacinados contra HPV." 2016. Disponível em <http://portalsaude.saude.gov.br/index.php/cidadao/principal/agenciasaude/25953-meninos-tambem-serao-vacinados-contra-hpv> Acesso em: 09 Março 2017.

BRASIL. Informe Técnico sobre a vacina Papiloma Vírus (HPV) na atenção básica. Brasília - $\quad$ Fevereiro, 2014.20 Disponível $<$ http://portalarquivos.saude.gov.br/images/pdf/2014/marco/07/Informe-T--cnico-vacinaHPV.pdf> Acesso em: 27 Março 2017.

BRASIL. PORTARIA No 3.212, DE 20 DE DEZEMBRO DE 2007. Disponível em <http://bvsms.saude.gov.br/bvs/saudelegis/gm/2007/prt3212_20_12_2007.html> Acesso em: 27 Março 2017.

BRASIL. Vacina contra HPV tem $98 \%$ de eficácia em quem segue cronograma. 2015 Disponível em <http://www.brasil.gov.br/saude/2015/03/vacina-contra-hpv-tem-98-deeficacia-em-quem-segue-cronograma> Acesso em: 19 Outubro 2017. 
CARNEIRO. R, F. et al. Educação sexual na adolescência: Uma abordagem no contexto escolar. Ano 2014 Disponível em <https://sanare.emnuvens.com.br/sanare/article/view/617/334> Acesso em: 26 Março 2017.

COELHO, N. Saúde amplia vacinação de HPV para homens e mulheres até 26 anos. 2017 Disponível em <http://portalsaude.saude.gov.br/index.php/cidadao/principal/agenciasaude/29280-saude-amplia-vacinacao-de-hpv-para-homens-e-mulheres-ate-26-anos> Acesso em: 25 Outubro 2017.

COELHO, N. Vacina de HPV é ampliada para meninos de 11 a 15 anos incompletos. 2017 Disponível em <http://portalsaude.saude.gov.br/index.php/cidadao/principal/agenciasaude/28769-vacina-de-hpv-e-ampliada-para-meninos-de-11-a-15-anos-incompletos-2>

Acesso em: 12 Outubro 2017.

DATASUS - Tecnologia da Informação a Serviço do SUS, 2016. Disponível em <http://tabnet.datasus.gov.br/cgi/tabcgi.exe?pni/CNV/CPNIBA.def> Acesso em: 8 Outubro 2017

IBGE - Instituto Brasileiro de Geografia e Estatísticas. Disponível em $<$ http://cidades.ibge.gov.br/xtras/perfil.php?lang=\&codmun=291200> Acesso em: 27 Maio 2017.

KAVATI, E. A. Desenvolvimento de vacina profilática e terapêutica contra o HPV e cânceres associados ao vírus. Plataforma Sucupira, São Paulo - SP 2017. Disponível em $<$ https://sucupira.capes.gov.br/sucupira/public/consultas/coleta/trabalhoConclusao/viewTrabal hoConclusao.jsf?popup=true\&id_trabalho=5021581> Acesso em: 12 Outubro 2017.

MACHADO, P. A. T.; ALCÂNTARA, A. C. M. Cobertura Vacinal Contra o HPV em Meninas de 09 a 11 Anos no Município de Rolim de Moura - RO. Revista Enfermagem e Saúde Coletiva, Faculdade São Paulo - FSP, 2016. Disponível em <http://www.revesc.org/index.php/revesc/article/view/2> Acesso em: 17 Maio 2017.

MINISTERIO DA SAÚDE. NOTA INFORMATIVA No $\mathbf{N}^{\circ}$ 62-SEI/2017CGPNI/DEVIT/SVS/MS. Disponível em <http://www.conasems.org.br/wpcontent/uploads/2017/08/NOTA-INFORMATIVA-N\%C2\%BA-62.pdf> Acesso em: 19 Outubro 2017.

OLIVEIRA, F. B.; GELATTI, L. C. Adesão das adolescentes frente á vacinação contra o HPV no município de Uruaçu, Goiás. 2015 Disponível em <www.fasem.edu.br/revista/index.php/fasemciencias/article/download/72/112> Acesso em: 25 Maio 2017.

QUEVEDO, J. et al. Implementação da vacina HPV no Brasil: Diferenciações entre a comunicação pública oficial e a imprensa midiática e sua relação com as coberturas vacinais. Revista Tecnológica e SociedadeR. Tecnol. Soc., Curitiba, v. 12, n. 24, p. 1-26, jan./abr. $2016 \quad$ Disponível em <http://www.rio2015.esocite.org/resources/anais/5/1440784582_ARQUIVO_Esocite2015.Va cinaHPVFinal.pdf> Acesso em: 27 Março 2017. 
SECRETÁRIA DA SAÚDE DO ESTADO DE GOIAS. Informe Técnico da Campanha de Vacinação do HPV e Meningo C. Goiânia 2017. Disponível em $<$ http://www.sgc.goias.gov.br/upload/arquivos/2017-08/informe-tecnico-campanha-na-escolada-vacinacao-do-hpv-e-meningo-c-19-07-17.pdf> Acesso em: 24 Outubro 2017.

SOUZA, S. E. B. Conhecimento e atitude de enfermeiros sobre câncer do colo do útero, infecção pelo Papiloma vírus Humano e vacinas contra o Papiloma vírus Humano. Fundação Oswaldo Cruz - Centro de pesquisas Gonçalo Muniz. Salvador - Bahia, 2015. Disponível

<https://www.arca.fiocruz.br/bitstream/icict/12238/2/Sandra\%20Ely\%20Barbosa\%20de\%20S ouza.\%20Conhecimento\%20e\%20atitude...pdf> Acesso em: 14 Maio 2017.

ZANINI, N. V. et al. Avaliação dos motivos de recusa á vacina contra o HPV no município de Maringá -PR. Anais Eletrônico IX EPCC - Encontro Internacional de Produção Científica UniCesumar, $2015 . \quad$ Disponível em <http://www.cesumar.br/prppge/pesquisa/epcc2015/anais/natalie_vieira_zanini_1.pdf> Acesso em: 18 Maio 2017.

Como citar este artigo (Formato ABNT):

PEREIRA, Fernanda de B.; SOUZA, Érika P. de. Cobertura Vacinal do HPV para Adolescentes: Desafios e Possibilidades. Id on Line Revista Multidisciplinar e de Psicologia, 2017, vol.11, n.38, p. 530-540. ISSN: 1981-1179.

Recebido: 01.10.2017

Aceito: 03.11.2017 